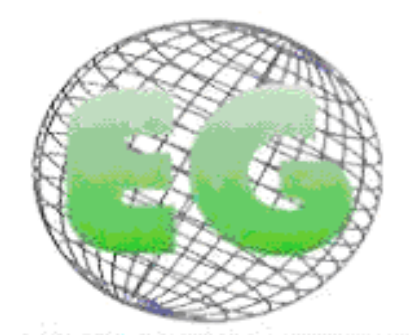

ISSN 1695-6141 $\mathrm{N}^{\circ} 27$

\title{
Autopercepción de la enfermedad en pacientes diagnosticados de diabetes mellitus tipo 2 que acuden a consulta de enfermería
}

Self-awareness of the disease in patients diagnosed as having type 2 diabetes mellitus attending nurse appointment

\section{*Martínez Castillo, Antonio.}

*Enfermero del Área de Gestión Sanitaria Norte de Almería. E-mail: amcastillo1981@gmail.com

Palabras clave: diabetes; autoidentificación; control metabólico.

Keywords: diabetes; self-identification; metabolic control

\section{RESUMEN}

Que el paciente se autoidentifique con la enfermedad que le ha sido diagnosticada y sea consciente de ella es fundamental para una correcta intervención sanitaria.

El objetivo del presente trabajo es conocer si los pacientes diabéticos se autoidentifican con su enfermedad y comprobar si esto influye en el control metabólico.

Material y método: Estudio descriptivo realizado a los pacientes diabéticos que acudieron a consulta de enfermería. Las variables fueron: autoidentificación como diabético, hemoglobina glicosilada, sexo, edad, situación laboral, nivel de estudios, convivir o no con pareja/cónyuge, años de diagnóstico y tipo de tratamiento. Se realizó análisis estadístico inferencial mediante los test de X2 y t de student.

Resultados: El $23,6 \%$ de la muestra no se autoidentifica con la diabetes. No existe significación estadística entre esta variable y las cifras de hemoglobina glicosilada. Sin embargo, sí existe relación con la edad y los años de diagnóstico. En el resto de variables tampoco se hallan datos significativos.

Conclusiones: Existe un alto porcentaje de pacientes que no son conscientes de su patología, algo que aumenta con la edad y disminuye con los años de diagnóstico. No existen diferencias en el control metabólico entre los que sí se autoidentifican y los que no.

\section{ABSTRACT}

In order to perform an appropriate health intervention, it is necessary that the patient is self-identified with the disease diagnosed.

The aim of this study is to know whether the diabetic patients are self-identified with this disease and to check whether this fact influences on the metabolic control or not. 
Material and Method: This is a descriptive study carried out on diabetic patients attending nurse appointment. The variables were the following: self-identification as a diabetic, glycosylated haemoglobin, gender, age, working situation, study level, living together or not, years of diagnosis, and kind of treatment. Inferencial statistical analysis performed using $\mathrm{X}^{2}$ and t-Student tests.

Results: $26,3 \%$ of the sample is not self-identified with diabetes. There is no statistical significance between this variable and the figures of glycosylated haemoglobin. However, there is a strong relationship between the age and the diagnosis years. No significant data is found in the other variables.

Conclusions: There is a high percentage of patients who are not aware of their pathology, this fact increments according to the age and decreases according to the diagnosis years. There are not any differences in relation to metabolic control between those who are self-identified and those who are not.

\section{INTRODUCCIÓN}

Que el paciente conozca su patología se presenta hoy día como un elemento clave en el proceso de la enfermedad, pues de esta manera la persona se siente más segura ante la misma. Pero antes de poder conocer la patología en sí, es necesario que la persona se reconozca a sí misma como enferma, ya que padece una determinada alteración en su estado de salud.

En este sentido, es necesario que los profesionales de enfermería conozcan qué actitudes tiene y qué respuestas está desarrollando el paciente crónico ante la enfermedad ${ }^{(1)}$. Para poder iniciar un plan terapéutico eficaz y conseguir una adecuada adherencia al tratamiento es imprescindible que la persona sea consciente de su estado de salud.

El hecho de no identificarse con la diabetes tiene una repercusión directa tanto en el control de la patología como en la calidad de vida. Es por esto por lo que conviene realizar una detección del problema lo más precoz posible, y de esta manera establecer estrategias de intervención que corrijan las actitudes de la persona. ${ }^{(2)}$

A groso modo, se pueden proponer dos razones por las que el paciente no se autoidentifique como diabético. Por un lado está la aceptación de la enfermedad; el paciente es conocedor del diagnóstico pero no lo acepta. De esta forma, el rechazo y la negación de la enfermedad suponen una de las dificultades que interfieren en el cumplimiento del tratamiento ${ }^{(3)}$ y por ende en el control metabólico de la patología. En un estudio relativo a este tema se demostró que entre el $77 \%$ y el $80 \%$ de los diabéticos (en función del hospital donde se realizó) no aceptaba su enfermedad. ${ }^{(4)}$

Por otro lado, cabe la posibilidad de que el paciente desconozca que está diagnosticado de diabetes, lo que sería una consecuencia a priori de una mala comunicación entre los profesionales sanitarios implicados y el usuario. Esta razón es muy poco mencionada por los autores, siendo prácticamente inexistentes los artículos que la mencionan.

En los últimos años está creciendo de forma considerable la tendencia de considerar al enfermo crónico como el protagonista activo de su patología para mejorar la intervención sobre el proceso salud-enfermedad ${ }^{(2)}$ pero es obvio que antes de que el paciente asuma las riendas es imprescindible que sea consciente de aquello que le sucede.

La idea de elaborar este estudio surge a raíz de la propia experiencia profesional, pues las situaciones anteriormente descritas se observan en el día a día de las consultas de enfermería y de forma habitual. 
Los objetivos propuestos para el presente trabajo son los siguientes:

- Conocer si las personas diagnosticadas de Diabetes Mellitus tipo 2, que acuden a consulta de enfermería, se autoidentifican como diabéticos y reconocen su enfermedad.

- Valorar si existen diferencias en los pacientes que asumen su enfermedad en el control metabólico, medido con la Hg glicosilada.

- Conocer la relación existente entre la autoidentificación de una persona como diabética y otra serie de variables.

\section{MATERIAL Y MÉTODOS}

\section{Diseño}

Se trata de un estudio observacional descriptivo realizado en el centro de salud de Macael perteneciente al Área de Gestión Sanitaria Norte de Almería del Servicio Andaluz de Salud. Este centro atiende a una población superior a los 6000 habitantes.

\section{Pacientes}

Para la selección de la muestra se utilizó a todas las personas diagnosticadas de Diabetes Mellitus tipo 2 que acudieron a la consulta de enfermería del centro de salud de la localidad desde el 1 de noviembre de 2010 al 31 de enero de 2011.

Los criterios de inclusión fueron:

- Aparecer diagnosticados de Diabetes Mellitus tipo 2 en el programa informático "Diraya" existente en el centro sanitario.

- Determinación de al menos una hemoglobina glicosilada (HbA1c) en el último año.

Y los criterios de exclusión:

- Personas que presenten un déficit cognitivo y enfermedad mental grave.

- Personas con idioma distinto al castellano.

\section{Variables de estudio}

Se recogieron las variables sexo, edad, situación laboral, nivel de estudios, convivir o no con pareja/cónyuge, años de diagnóstico de la diabetes, tipo de tratamiento, comorbilidad, cifras de hemoglobina glicosilada y autoidentificación como diabético.

La recogida de los datos se realizó mediante entrevista personal y a través del programa informático "Diraya" existente en el centro y de la base de datos del servicio de laboratorio del Área de Gestión Sanitaria Norte de Almería.

Para recoger información sobre la variable "autoidentificación como diabético" se les realizó la pregunta ¿Es usted diabético? durante la entrevista clínica. La persona encargada de la recogida de datos fue siempre la misma para evitar los sesgos derivados del profesional que lo realizaba. 


\section{Análisis estadístico}

Para el análisis estadístico se utilizó el soporte informático SPSS para Windows en su versión 17. Las variables cuantitativas se describieron mediante media, desviación típica, valor máximo, valor mínimo y rango. Por otro lado, se utilizaron frecuencias relativas en porcentajes para las variables cualitativas. Las relaciones entre variables se analizaron con el test de $\mathrm{X}^{2}$ para variables cualitativas y el test paramétrico $t$ de Student para la comparación de medias.

\section{RESULTADOS}

La muestra total del estudio fueron 89 pacientes, con una edad media de 69,08 años y el $52,8 \%$ eran mujeres.

No se autoidentificaron como diabéticos el $23,6 \%$ de los pacientes y la cifra de hemoglobina glicosilada (HbA1c) media del total de personas fue del 7,327\%.

En las tablas I y II se describen todas las variables estudiadas.

\section{Tabla I. Variables cuantitativas.}

\begin{tabular}{|c|c|c|c|c|c|}
\hline & Media & Desv. Típ. & Rango & Valor mínimo & Valor máximo \\
\hline HbA1c & 7,327 & 1,4625 & 8,5 & 4,6 & 13,1 \\
\hline Edad & 69,08 & 9,96 & 40 & 46 & 86 \\
\hline $\begin{array}{c}\text { Años de } \\
\text { diagnóstico }\end{array}$ & 6,75 & 5,166 & 20 & 0 & 20 \\
\hline \begin{tabular}{c} 
Comorbilidad \\
\hline
\end{tabular} & 2,35 & 1,332 & 6 & 0 & 6 \\
\hline
\end{tabular}




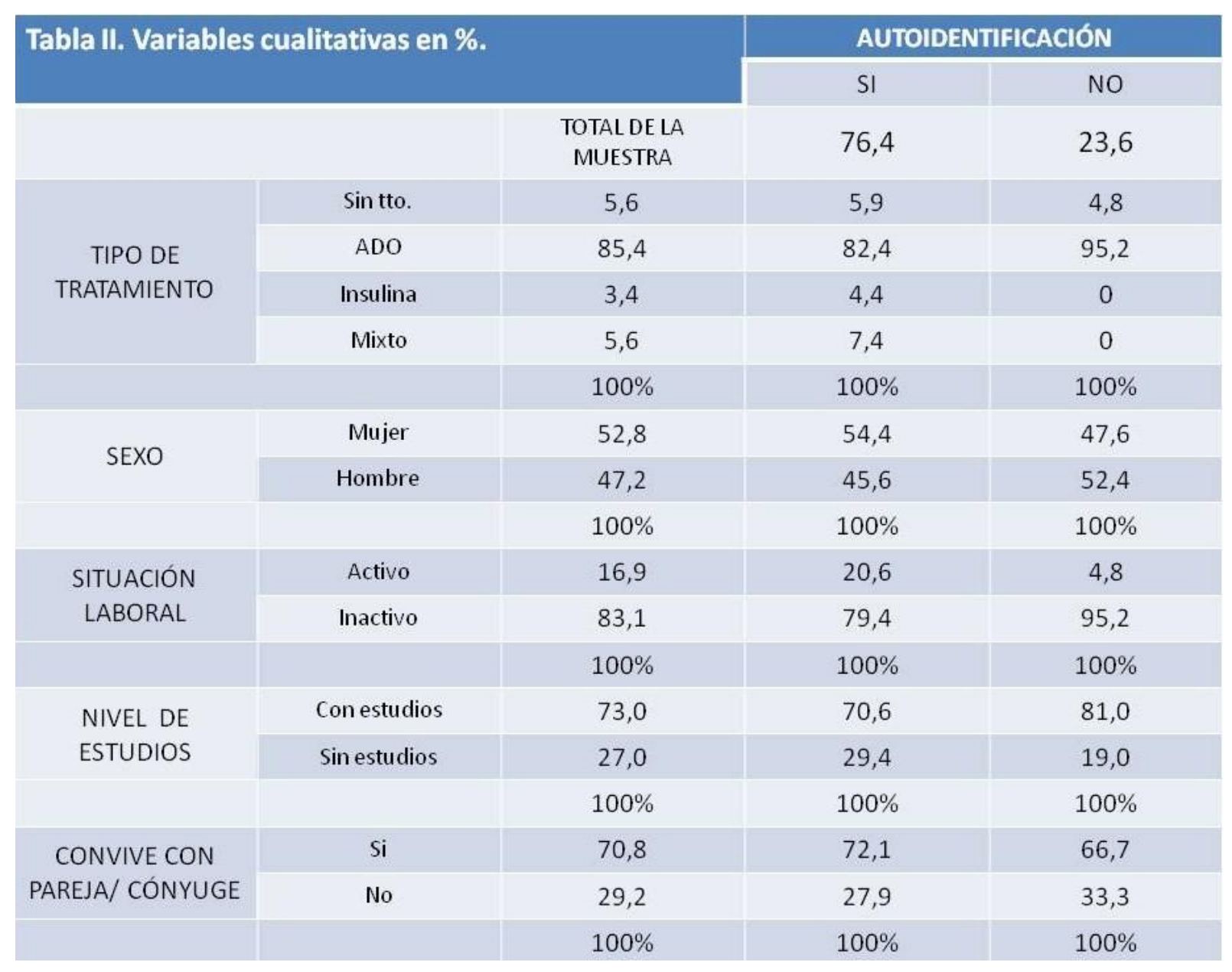

\section{Autoidentificación como diabéticos y control metabólico}

Las cifras de $\mathrm{HbA} 1 \mathrm{c}$ fueron del $7,385 \%$ entre aquellos que sí se autoidentificaban como diabéticos y del $7,138 \%$ entre los que no. No se estableció asociación significativa entre ambas variables $(p<0,05)$, con una $p=0,654$ obtenida mediante t de Student y no asumiendo varianzas iguales tras aplicar la prueba de Levene para igualdad de varianzas.

\section{Autoidentificación como diabéticos y años de diagnóstico}

Las personas que se consideraban diabéticas tenían una media de 7,47 años desde que se les diagnosticó la enfermedad y de 4,43 años aquellos pacientes que no asumían su enfermedad. Se observó una significación estadística relacionando ambas variables [t de student $p=0,008(p<0,05)$, no asumiendo varianzas iguales tras realizar la prueba de Levene].

\section{Autoidentificación como diabéticos y edad}

La media de edad de las personas que se autoidentificaban con la enfermedad $(67,87$ años $)$ era menor que la media de las que no se autoidentificaban (73 años). Además se observó significación estadística mediante $t$ de student obteniendo una $p=0,038 \quad(p<0,05)$, asumiendo varianzas iguales tras la realización de la prueba de Levene. 


\section{Autoidentificación como diabéticos y comorbilidad}

La comorbilidad media de las personas que asumen la diabetes era de 2,29 y la de aquellos que no la asumen era de 2,52. Además, para entender mejor esta variable se estableció una estratificación de la comorbilidad como se puede apreciar en la tabla III. Por otro lado, con una $p=0,545$ con el test $t$ de Student $(p<0,05)$, se pudo determinar la no existencia de asociación significativa entre ambas variables, no asumiendo varianzas iguales.

\begin{tabular}{|c|c|c|c|}
\hline & Total de la muestra & $\begin{array}{c}\text { SI } \\
\text { autoidentificación }\end{array}$ & $\begin{array}{c}\text { NO } \\
\text { autoidentificación }\end{array}$ \\
\hline No comorbilidad & 5,6 & 7,3 & 0 \\
\hline 1-2 patologías & 56,2 & 55,9 & 57,1 \\
\hline 3 o más patologías & 38,2 & 36,8 & 42,9 \\
\hline
\end{tabular}

\section{Autoidentificación como diabéticos y tipo de tratamiento}

Como se puede observar en la tabla II, existen dos casillas en las que el porcentaje (y por ello el número de sujetos incluidos en la misma) es igual a 0,0 por lo que el análisis estadístico inferencial no fue posible.

\section{Otras variables}

Con el fin de sintetizar y expresar más gráficamente el resto de variables estudiadas se creó la siguiente tabla (tabla IV) donde se describieron las pruebas estadísticas utilizadas y el grado de asociación de las variables con respecto a la autoidentificación como diabético $(p<0,05)$.

Tabla IV. Otras variables estudiadas

\begin{tabular}{cccc|}
\hline VARIABLE & PRUEBA & $p$ & \\
\hline Sexo & $\chi^{2}$ Yates & 0,768 & $\begin{array}{c}\text { No asociación } \\
\text { significativa }\end{array}$ \\
$\begin{array}{c}\text { Situación } \\
\text { laboral }\end{array}$ & $\chi^{2}$ Fisher & 0,108 & $\begin{array}{c}\text { No asociación } \\
\text { significativa }\end{array}$ \\
\hline $\begin{array}{c}\text { Nivel estudios } \\
\text { Convivencia con }\end{array}$ & $\chi^{2}$ Yates & 0,513 & $\begin{array}{c}\text { No asociación } \\
\text { significativa }\end{array}$ \\
\hline pareja/cónyuge & & 0,841 & $\begin{array}{c}\text { No asociación } \\
\text { significativa }\end{array}$ \\
\hline
\end{tabular}




\section{DISCUSIÓN}

En primer lugar cabe destacar tres limitaciones del presente trabajo. Por un lado, el hecho de incluir únicamente en la muestra a las personas que acuden a consulta hace que se pierdan aquellos que apenas utilizan los servicios sanitarios y que se presupone que pueden estar peor controlados. Por otro lado encontramos que el tamaño de la muestra es mucho menor al que en principio se intuía ya que fueron muchos los pacientes que no tenían realizada una hemoglobina glicosilada en el último año y tuvieron que ser desechados, tal y como se especifica en los criterios de inclusión. Por último, cabe destacar que en un principio se diseñó el estudio con un muestreo probabilístico sistemático, pero debido a la escasez de pacientes por la razón mencionada se determinó finalmente incluir a todos los pacientes que acudían a consulta sin aleatoriedad alguna.

También debido al tamaño muestral, a la hora de realizar el análisis estadístico de los datos se hizo necesaria la redefinición de algunas variables cualitativas, teniendo que agrupar y reducir el número de categorías posibles de cada variable, pues el número de sujetos incluidos en cada categoría hacía que los test estadísticos no se pudieran aplicar. Por ello, la variable "situación laboral" que en principio podía adoptar cinco categorías (activo, incapacidad temporal, paro, jubilado por edad y jubilado por incapacidad) se redujo a dos (activo e inactivo). También la variable "nivel de estudios" con cuatro categorías (sin estudios, primarios, secundarios y superiores) se modificó a dos (con estudios y sin estudios). Además, la variable definida en primer lugar como "estado civil" (categorías: casado, viudo, soltero y divorciado) tuvo que ser redefinida como "convivencia con pareja/cónyuge" y adoptar dos posibles categorías (convive y no convive). Sin embargo, para la variable "tipo de tratamiento" no se consideró oportuna la reagrupación, prefiriendo descartarla del análisis estadístico inferencial.

El hecho de utilizar en este trabajo como medida de referencia la hemoglobina glicosilada se debe a que es considerada como el mejor parámetro de control glucémico en la diabetes. ${ }^{(5,6)}$ El presente estudio nos aporta como principal resultado un porcentaje importante $(23,6 \%)$ de personas que no saben 0 no son conscientes de su enfermedad, pese a estar diagnosticadas de diabetes mellitus por su médico y a tener este diagnóstico reflejado en su historia clínica. En contraposición a este dato encontramos las teorías ya mencionadas en la introducción, por las cuales se considera al enfermo crónico como el protagonista activo de su patología, favoreciendo de esta forma la intervención en el proceso de saludenfermedad. ${ }^{(2,7,8)}$ Pero ¿cómo el paciente puede ser protagonista de su enfermedad si ni siquiera sabe que está enfermo? Encontramos en este punto un posible problema de comunicación entre el profesional sanitario (ya sea personal de enfermería o de medicina) y el paciente. La educación diabetológica se plantea como una de las principales estrategias para conseguir un correcto control metabólico y de la diabetes en sí, entonces, sería fundamental que uno de los objetivos de esta estrategia esté encaminado a corregir este punto tan importante. Respecto a esto hay que destacar que el proceso educativo no debe limitarse a una mera transmisión de conocimientos, sino que debe englobar aspectos emocionales, sociales y culturales $^{(3)}$ que puedan determinar la interpretación y el seguimiento de la enfermedad.

Obtenemos en nuestra muestra una $\mathrm{HbA1c}$ media del $7,327 \%$, resultado que no difiere en exceso con las cifras obtenidas en el estudio realizado por Mena Martín et al, ${ }^{(9)}$ donde la media se situaba en $7,8 \%$. En vista de los resultados obtenidos tras el análisis estadístico que relaciona la autoidentificación como diabético y las cifras de HbA1c podemos afirmar que no están mejor controlados metabólicamente los pacientes que son conscientes de su enfermedad, como se puede pensar en un principio. Tampoco están peor controlados 
aquellos que no se identifican como diabéticos. A partir de estas afirmaciones se pueden barajar dos posibles hipótesis: Que tanto unos como otros se cuidan igual de bien, por lo que están igualmente controlados, o que ambos grupos se cuidan igual de mal y por ello no existen diferencias significativas en el grado de control. Además de abrir un nuevo campo de investigación, estos resultados han de servir para la reflexión profunda de los profesionales sanitarios implicados.

En cuanto a los resultados del análisis inferencial entre las variables autoidentificación como diabético y edad/años de diagnóstico de los pacientes, encontramos que sí existe significación estadística, por lo que se puede afirmar que los pacientes más longevos reconocen peor su enfermedad que los pacientes menos longevos y que aquellos con más años desde que fueron diagnosticados se autoidentifican con esta enfermedad mejor que los pacientes de más reciente diagnóstico.

Como se expresa en el apartado anterior, no fue posible analizar la correlación existente con el tipo de tratamiento empleado por la persona. Sin embargo merece especial mención el hecho de que no existan pacientes que se inyecten insulina y que no se autoidentifiquen con la diabetes. En este sentido, Moreno C, et al. ${ }^{(10)}$ concluyen que la insulinización hace que aumente la percepción de gravedad de la diabetes, afirmando que los diabéticos que no son insulinodependientes se sienten "menos diabéticos" que los que sí precisan insulina.

Por último, encontramos que en el resto de variables analizadas no se ha hallado significación estadística alguna. Esto puede deberse al reducido tamaño muestral, por lo que son necesarias nuevas investigaciones en las que el número de individuos sea superior.

\section{CONCLUSIONES}

- Existe un alto porcentaje de diabéticos que no se autoidentifican con la enfermedad que padecen.

- No existe relación entre autoidentificarse o no como diabético y el grado de control de la enfermedad, medido mediante la hemoglobina glicosilada.

- Las personas de menor edad se auoidentifican más con la diabetes que las personas de mayor edad.

- Los pacientes con más años desde el diagnóstico se autoidentifican mejor con la diabetes que los pacientes de más reciente diagnóstico.

- No existe relación alguna en nuestra muestra con el resto de variables estudiadas.

\section{BIBLIOGRAFÍA}

1.Morillas Herrera J, Martín Santos F. Consulta de enfermería para pacientes crónicos. Rev. Rol de enfermería 2001; 24(6): 467-471.

2. Bolaños E, Sarriá-Santamera A. Perspectiva de los pacientes sobre la diabetes tipo 2 y relación con los profesionales sanitarios de atención primaria: un estudio cualitativo. Aten Primaria 2003; 32(4): 195-202.

3. Peres D, Santos M, Zanetti M, Ferronato A. Dificultades de los pacientes diabéticos para el control de la enfermedad: sentimientos y comportamientos. Rev. Latino-Am. Enfermagem 2007; 15(6): 1105-1112.

4. Saavedra Martínez D. Paciente diabético; ¿conoce y acepta su padecimiento? Rev Enferm IMSS 2001; 9(1): 5-7. 
5. De la Calle H, Costa A, Díez-Espino J, Franch J, Goday A. Evaluación del cumplimiento de los objetivos de control metabólico de la diabetes mellitus tipo 2. Estudio tranSTAR. Med Clin 2003; 120:446-450.

6. American Diabetes Association. Standards of medical care for patients with diabetes mellitus. Diabetes Care 2002;25(Suppl 1):33-49.

7. Kinmonth AL, Spiegal N,Woodcock A. Developing a training programme in patientcentred consulting for evaluation in a randomized controlled trial; diabetes care from diagnosis in British primary care. Patient Educ Couns 1996; 29:75-86.

8. Stewart M, Brown JB, Boon H, Galajda J, Meredith L, Sangster M. Evidence on patientdoctor communication. Cancer Prev Control 1999; 3:25-30.

9. Mena F, Martín J, Simal F, Bellido J, Carretero J. Diabetes mellitus tipo 2 y calidad de vida relacionada con la salud: resultados del Estudio Hortega. An. Med. Interna 2006; 23. En: http://dx.doi.org/10.4321/S0212-71992006000800002

10. Moreno $\mathrm{C}$, et al. Creencias, actitudes y conocimientos de las personas con diabetes tipo 2 respecto a su enfermedad. Enfermería clínica 2004; 14(6): 307-312. 